\title{
Higher expression of epidermal growth factor receptor is associated with extracellular matrix metalloprotease inducer in colorectal adenocarcinoma: Tissue microarray analysis of immunostaining score with clinicopathological parameters
}

\author{
Jong-Shiaw Jin ${ }^{\mathrm{a}}$, Chi-Ying $\mathrm{Wu}^{\mathrm{a}}$, Yeh-Feng Lin ${ }^{\mathrm{b}}$, Jia-Yi Wang ${ }^{\mathrm{c}}$, Cheng-Ping Yu ${ }^{\mathrm{a}}$, Lai-Fa Sheu ${ }^{\mathrm{a}}$, \\ Hung Chiang ${ }^{\mathrm{d}}$, Wen-Chiuan Tsai $^{\mathrm{a}}$ and Wei-Hwa Lee ${ }^{\mathrm{a}, *}$ \\ ${ }^{a}$ Department of Pathology, Tri-Service General Hospital \\ ${ }^{\mathrm{b}}$ Division of Nephrology, Department of Internal Medicine, Tri-Service General Hospital \\ ${ }^{\mathrm{c}}$ Department of Physiology, Tri-Service General Hospital, National Defense Medical Center, Taipei, Taiwan \\ ${ }^{\mathrm{d}}$ Taipei Institute of Pathology, Taipei, Taiwan
}

\begin{abstract}
Aim: Extracellular matrix metalloprotease inducer (EMMPRIN) expression was demonstrated in several cancers, but its expression profile in colorectal cancers remains unclear. Epidermal growth factor receptor (EGFR) was reported to regulate EMMPRIN expression in human epithelial cancers. Our purpose was to determine EMMPRIN expression and its relationship with EGFR in colorectal cancers.

Methods: Immunohistochemical analysis of EMMPRIN and EGFR was performed in tissue microarray slides of 90 surgical specimens including 32 well differentiated, 35 moderately differentiated, and 23 poorly differentiated colorectal adenocarcinomas. Results: All colorectal adenocarcinomas showed significant immunohistochemical expression of EMMPRIN. The EMMPRIN scores in poorly differentiated $(303 \pm 21)$ and moderately differentiated $(326 \pm 17)$ colorectal adenocarcinoma were significantly higher than in well differentiated $(166 \pm 20)$ colorectal adenocarcinoma. EGFR expression was mainly on the cell surface of tumor cells and the immunostaining scores of EGFR were significantly associated with the advanced clinical T and N stages. A significantly positive relationship between EMMPRIN and EGFR immunostaining scores was also noted.

Conclusions: Increased expression of EMMPRIN and EGFR in colorectal adenocarcinomas is associated with clinicopathological parameters of advanced colorectal adenocarcinoma stages. In addition, the data from this study support the notion that EGFR expression may up-regulate EMMPRIN expression.
\end{abstract}

Keywords: Extracellular matrix metalloprotease inducer, EMMPRIN, epidermal growth factor receptor, colorectal, adenocarcinoma

* Corresponding author: Wei-Hwa Lee, M.D., Ph.D., Department of Pathology, Tri-Service General Hospital, National Defense Medical Center, No. 325, Sec. 2, Cheng-Gong Road, Taipei, Taiwan,
R.O.C., Tel.: +886 2 87923311, Ext. 16736; Fax: +8862 26913324; E-mail: jsjin@ndmctsgh.edu.tw. 


\section{Introduction}

Extracellular matrix metalloproteinase inducer (EMMPRIN/CD147) is a membrane spanning glycoprotein that belongs to the immunoglobulin superfamily and plays a role in neoplastic metastasis $[7,10]$. The expression of EMMPRIN in tumor cells [13] results in the upregulation of matrix metalloproteinase (MMPs) at the epithelio-stromal interface $[17,20,25]$. Studies have shown that the expression of MMPs by stromal fibroblasts in most tumors is correlated with EMMPRIN expression [20,25]. The EMMPRIN expression has been demonstrated in certain cancers including breast, ovary, oral, larynx, esophagus, and lung cancers. However, EMMPRIN expression in colorectal adenocarcinoma is still unclear.

Epidermal growth factor receptor (EGFR) is a cell surface protein that is commonly found in epithelial cancers $[3,9,12]$. A prior study showed that the induction of EMMPRIN in human breast epithelial cells lines is influenced by EGFR activation [26]. To clarify the expression profile of EMMPRIN and its relationship to EGFR expression, we evaluated the expression profiles of EMMPRIN and EGFR in surgical specimens of 90 colorectal adenocarcinomas using tissue microarray, and compared immunostaining scores for EMMPRIN and EGFR between different histopathological types of colon cancer.

\section{Materials and methods}

Paraffin-embedded tissue blocks of resected tumor specimens were retrieved from the Department of Pathology, Tri-Service General Hospital. The entired tumor sections of 90 primary colorectal adenocarcinomas were evaluated and classified including 32 well differentiated (glandular structure $>95 \%$ ), 35 moderately differentiated (glandular structure between 50\%$95 \%$ ), and 23 poorly differentiated tumors (glandular structure $<50 \%$ ). The histopathological differentiation of the colorectal adenocarcinoma was determined according to the criteria of WHO classification of tumor. Although the selected tumors in this study often showed heterogeneity in differentiation and had variation in expression of EMMPRIN and EGFR, this variation was minimized by increasing the case number to more than 60 cases (8). Thus, the probability that results from one core would correctly represent the whole section is more than $91 \%$ (8).
Table 1

Immunostaining scores of EMMPRIN in colorectal adenocarcinoma

Differentiation

Tumor

$\begin{array}{lccc} & \text { Intensity } & \% \text { staining } & \text { Total score } \\ \text { Well }(n=32) & 2.2 \pm 0.3^{*} & 71 \pm 6^{*} & 166 \pm 20^{*} \\ \text { Moderate }(n=35) & 3.3 \pm 0.2^{*} & 98 \pm 1^{*} & 326 \pm 17^{*} \\ \text { Poor }(n=23) & 3.1 \pm 0.2^{*} & 95 \pm 4^{*} & 303 \pm 21^{*}\end{array}$

Poor $(n=23) \quad 3.1 \pm 0.2 * \quad 95 \pm 4^{*} \quad 303 \pm 21^{*}$

Normal epithelia $(n=9) \quad 1.5 \pm 0.3 \quad 15 \pm 7 \quad 23 \pm 14$

Data are means \pm standard error of the mean (SEM) of immunostaining scores for EMMPRIN in colorectal adenocarcinoma. * indicates significant difference of EMMPRIN expression between tumor and normal colorectal epithelia $(P<0.05)$.

Table 2

Immunostaining scores for EGFR in colorectal adenocarcinoma

\begin{tabular}{lccc} 
Differentiation & \multicolumn{3}{c}{ Tumor } \\
& Intensity & $\%$ staining & Total score \\
Well $(n=32)$ & $1.6 \pm 0.2^{*}$ & $58 \pm 8^{*}$ & $132 \pm 22^{*}$ \\
Moderate $(n=35)$ & $1.8 \pm 0.2^{*}$ & $76 \pm 6^{*}$ & $164 \pm 21^{*}$ \\
Poor $(n=23)$ & $2.0 \pm 0.2^{*}$ & $72 \pm 8^{*}$ & $159 \pm 24^{*}$ \\
Normal epithelia & $0.8 \pm 0.3$ & $25 \pm 6$ & $48 \pm 17$
\end{tabular}

Data are means \pm standard error of the mean (SEM) of immunostaining score for EGFR in colorectal adenocarcinoma. * indicates significant difference of EGFR expression between tumor and normal colorectal epithelia $(P<0.05)$.

One core was taken from a selected area of each paraffin-embedded tumor tissue and tissue microarray slides were constructed according to previously published methods $[2,14,16]$. Each representative core in the tissue microarray slide was $2 \mathrm{~mm}$ in diameter and the pathological diagnosis in these cases was reviewed by at least two experienced pathologists. All the colorectal adenocarcinoma cases were classified on the basis of histological grading and TNM staging. All tumors were pathologically staged according to the 1997 AJCC/TNM system. Normal colon tissues from nine cases (mean age 54) that included columnar epithelia were obtained; tissues were taken at least $4 \mathrm{~cm}$ from the neoplasm.

\subsection{Immunohistochemistry}

Tissue microarray sections were dewaxed in xylene, rehydrated in alcohol, and underwent antigen retrieval $\left(0.01 \mathrm{~mol} / \mathrm{L}\right.$ sodium citrate buffer $(\mathrm{pH} 6.0)$ at $100^{\circ} \mathrm{C}$ for $30 \mathrm{~min}$ ) and endogenous peroxidase activity suppression (immersion in 3\% hydrogen peroxide for $5 \mathrm{~min}$ ). After 3 rinses (each for $5 \mathrm{~min}$ ) in phosphate buffered saline (PBS), sections were incubated for $1 \mathrm{~h}$ at room temperature with a mouse anti-human EMMPRIN antibody (1:100, Santa Cruz Biotechnology, Inc., Santa Cruz, CA, USA), or mouse anti-human EGFR antibody (1:50, Neomarker Inc., Freemont, CA, USA) diluted in 


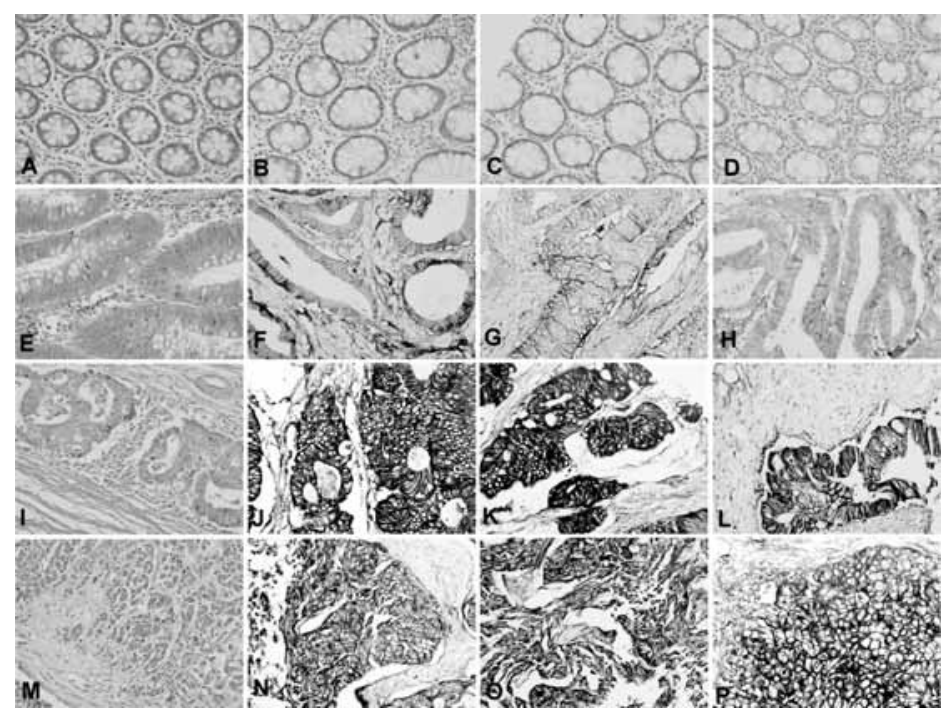

Fig. 1. Hematoxylin and eosin staining of non-neoplastic colorectal epithelia (A), well differentiated (E), moderately differentiated (I), and poorly differentiated (M) colorectal adenocarcinoma. Immunohistochemical analysis of EMMPRIN in non-neoplastic colorectal epithelia (B, score 17; C, score 30; and D, score 20), well differentiated (F, score 150; G, score 180; and H, socre 170), moderately differentiated (J, score 300; K, score 350; and L, score 340), and poorly differentiated ( $\mathrm{N}$, score 280; O, score 300; and $\mathrm{P}$, score 330) colorectal adenocarcinoma. Original magnification X 400 .

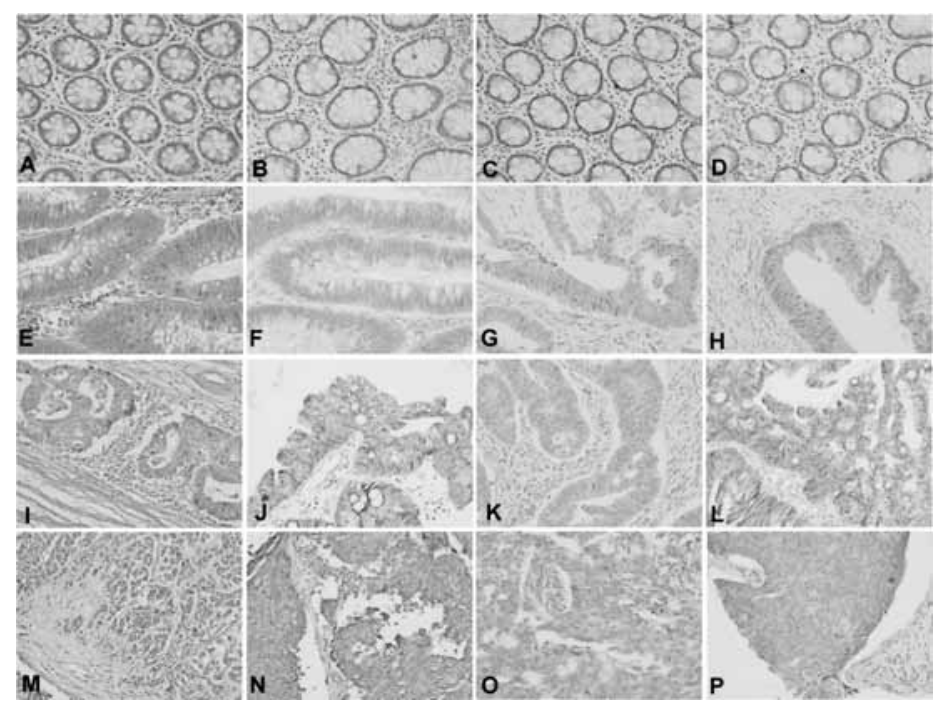

Fig. 2. Hematoxylin and eosin staining of non-neoplastic colorectal epithelia (A), well differentiated (E), moderately differentiated (I), and poorly differentiated (M) colorectal adenocarcinoma. Immunohistochemical analysis of EGFR in non-neoplastic colorectal epithelia (B, score 30; C, score 50; and D, score 45), well differentiated (F, score 140; G, score 150; and H, socre 130), moderately differentiated (J, score 150; $\mathrm{K}$, score 170; and L, score 180), and poorly differentiated (N, score 170; O, score 140; and P, score 180) colorectal adenocarcinoma. Original magnification X 400 .

PBS. After 3 washes (each for $5 \mathrm{~min}$ ) in PBS, the sections were incubated with the biotinylated secondary antibody (1:100, Dako, Glostrup, Denmark) for $40 \mathrm{~min}$. After 3 rinses in PBS, horseradish-peroxidase conjugated streptavidin was added for $20 \mathrm{~min}$ at room temperature. The peroxidase activity was developed with the chromogen DBA (Dako) for $15 \mathrm{~min}$ at room temperature and then counterstained with Meyer's hematoxylin.

For evaluation of immunoreactivity and histological appearance, tissue microarray slides were examined and scored by two authors concurrently, and the av- 
erage immunostaining scores of two pathologists were recorded. If discrepancy (in 3 cases) occurred, the third pathologist's opinion was included. Slides with more than $50 \%$ of the tissue detached during the immunohistochemistry process were not scored. The intensity of membranous and cytoplasmic immunostaining of EMMPRIN was scored on a scale of 0 (no staining) to 4 (strongest intensity), and the percentage of cells at each staining intensity was estimated. The percentage of cells at each intensity ( 0 to 100$)$ was multiplied by the corresponding intensity value ( 0 to 4 ), and these products were added to obtain an immunostaining score ranging from 0 to 400 .

\subsection{Statistical analysis}

All results are expressed as mean \pm standard error of the mean (SEM). The immunostaining scores of EMMPRIN and EGFR of colorectal adenocarcinoma were compared with the score of normal colon epithelia. Statistical analysis was performed using the Student $t$-test between groups. A $\mathrm{P}$ value less than 0.05 was considered to be statistically significant. Oneway analysis of variance (ANOVA) was used to determine statistical significance among different types of colon cancer. SigmaState software (Jandel Scientific, San Rafael, CA, USA) was used to perform linear regression testing to analyze the relationship between EMMPRIN or EGFR immunostaining score and clinicopathological parameters.

\section{Results}

\subsection{EMMPRIN expression in colorectal adenocarcinoma}

Tissue specimens from 32 well differentiated, 35 moderately differentiated and 23 poorly differentiated colorectal adenocarcinomas were scored for EMMPRIN. The intensity and percentage of positively immunostained cells in the colon adenocarcinoma are shown in Table 1 and Fig. 1. Significant increase immunostaining scores of EMMPRIN were noted in colon adenocarcinoma when compared to non-tumor epithelia (166 \pm 20 for well differentiated, $326 \pm 17$ for moderately differentiated, and $303 \pm 21$ for poorly differentiated adenocarcinomas, Fig. 1 and Table 1).
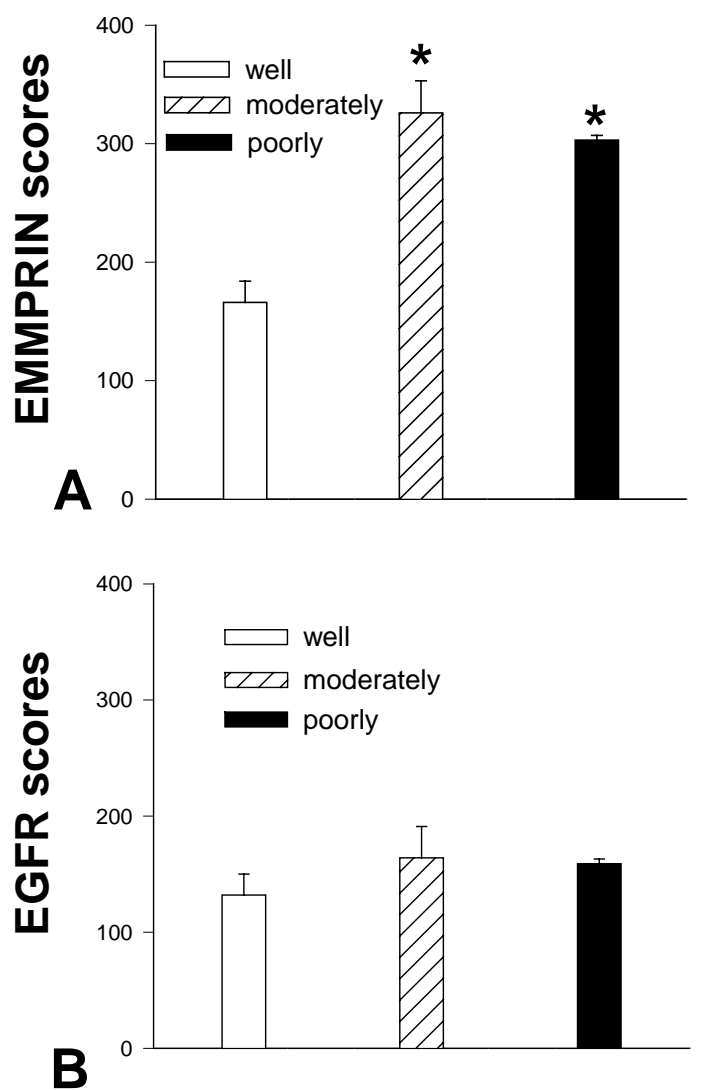

Fig. 3. Immunostaining scores of EMMPRIN (A) and EGFR (B) in well differentiated, moderately differentiated, and poorly differentiated colorectal adenocarcinoma. * indicates statistical significance comparing to well differentiated colorectal adenocarcinoma $(p<0.05)$.

\subsection{EGFR expression in colorectal adenocarcinoma}

Specimens from 32 well differentiated, 35 moderately differentiated, and 23 poorly differentiated colorectal adenocarcinomas were scored for EGFR (Table 2 and Fig. 2). Significantly increased EGFR immunostaining score was noted in colon adenocarcinoma when compared to non-tumor epithelia (132 \pm 22 for well differentiated, $164 \pm 21$ for moderately differentiated, and $159 \pm 24$ for poorly differentiated adenocarcinomas).

The immunostaining scores of EMMPRIN were significantly higher in moderately differentiated and poorly differentiated colorectal adenocarcinoma compared to well differentiated colorectal adenocarcinomas (Fig. 3A). There was no significant difference in EGFR immunostaining score between different histopathological differentiations of colorectal adenocarcinoma (Fig. 3B). 


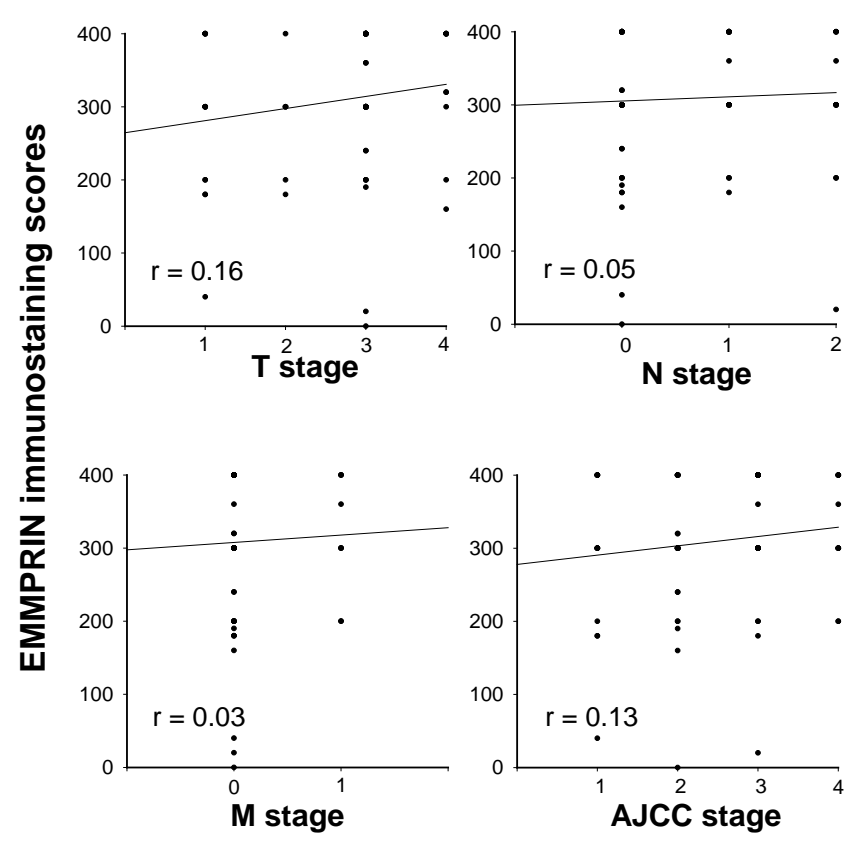

Fig. 4. Clinicopathological correlations with EMMPRIN immunostaining scores in colorectal adenocarcinoma. No statistical significance of linear regression testing was reached.

\subsection{Clinicopathological association}

Linear regression testing to determine the relationship between the immunostaining scores of EMMPRIN and the clinical TNM stages of tumors found no significant association between the immunostaining scores of EMMPRIN and clinical TNM stages (Fig. 4).

Linear regression testing was performed to analyze the relationship between the EGFR immunostaining score and TNM stage. The EGFR immunostaining score was significantly positively correlated with the $\mathrm{T}$ and nodal (N) stages but not with the M stage (Fig. 5).

\subsection{Correlation between EGFR and EMMPRIN expression}

The correlation between EGFR immunostaining scores and EMMPRIN immunostaining scores is shown in Fig. 6. Significantly higher EGFR immunostaining score was associated with elevated EMMPRIN immunostaining score in colorectal adenocarcinoma.

\section{Discussion}

We have demonstrated for the first time that EMMPRIN and EGFR are over-expressed in colorectal adenocarcinomas. Increased expression of EMMPRIN and EGFR in colorectal adenocarcinomas is associated with clinicopathological parameters of advanced colorectal cancer stages. In addition, increasing EGFR expression may up-regulate EMMPRIN expression in colorectal adenocarcinoma.

Our current study was designed using tissue microarray. One potential limitation of tissue microarray is the correct representation of each tumor with the level of heterogeneity. However, a study has demonstrated that when the number of cases is increase to more than 54 cases in tissue microarray preparation, the probability that results from one core would correctly represent the whole section was more than $91 \%$ [8]. In previous studies, immunohistochemical quantitation varied between cases because immunostaining intensity was affected by changes in environmental or amplification conditions. The tissue microarray technique eliminated this problem by permitting simultaneous analysis of all tumor tissue samples on the same slide [21]. Thus, a large number of samples assayed under the same conditions in a single tissue microarray could be evaluated in a quantitative fashion $[2,14,16,23]$ and more reliably than individual tissues assayed one at a time.

The EGFR is a family of four cell membrane receptors $($ Erb1 Erb4) [4]. Each of these cell surface proteins has an extracellular ligand binding domain, a transmembrane domain, and an internal domain with tyrosine kinase activity [4]. Activation by ligands (such 


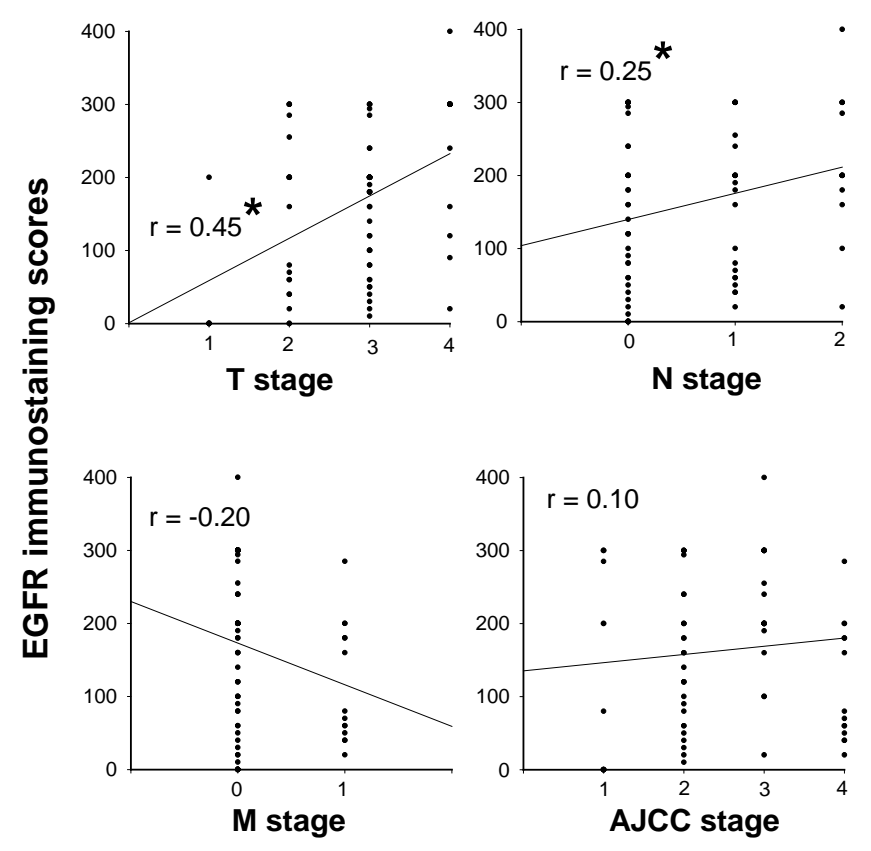

Fig. 5. Clinicopathological correlations with EGFR immunostaining scores in colorectal adenocarcinoma. * indicates statistical significance of linear regression testing $(p<0.05)$.

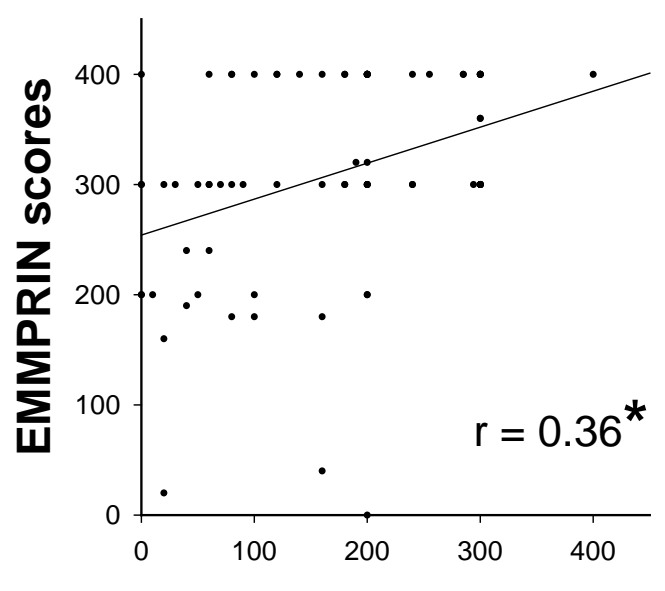

EGFR scores

Fig. 6. Correlation of EGFR immunostaining scores with EMMPRIN immunostaining scores in colorectal adenocarcinoma. * indicates statistical significance of linear regression testing $(p<0.05)$.

as epidermal growth factor) leads to EGFR dimerization and autophosphorylation, initiating a cascade of cellular events known as the signal transduction pathway $[1,4]$. This process results in increased rate of cell division and influences other aspects of malignant progression [15].

A previous study showed that expression of EMMPRIN was up-regulated by EGFR activation, and down- regulated by antisense EGFR RNA in human breast epithelial cells [26]. Overexpression or autoactivation of EGFR in tumor cells may induce the expression of EMMPRIN which subsequently stimulates the production of matrix metalloproteinases and thereby tumor progression and metastasis $[10,20,25]$. Recently, the EGFR tyrosine kinase inhibitors and monoclonal antibodies against EGFR were developed as new targeted anti-cancer agents for treatment of metastatic colorectal adenocarcinoma [15,24]. In the present study, higher EGFR immunostaining scores were positively correlated with EMMPRIN immunostaining scores in colorectal adenocarcinoma. Our findings are consistent with the conclusion that EGFR contributes to the molecular pathogenesis of colorectal cancer by increasing cancer cell invasion through up-regulation of EMMPRIN.

EMMPRIN is expressed in breast cancer [5,22], Tcell lymphoma [19], ovarian cancer [6], laryngeal cancers [11], esophageal squamous cell carcinoma [28], lung cancers [27] and bladder cancers [18]. In the present study, EMMPRIN was widely expressed on the cell surface of colorectal adenocarcinoma cells, minimally expressed in normal colorectal epithelia, and significantly more expressed in advanced histopathological grade (moderately and poorly differentiated) colorectal adenocarcinomas. Therefore, EMMPRIN might play a role in colorectal adenocarcinoma pro- 
gression to advanced histolopathological grade. More effort will be directed at elucidating its role in tumor dedifferentiation and progression.

In conclusion, we demonstrate for the first time that EMMPRIN is overexpressed in colorectal adencarcinomas. Increasing expression of EMMPRIN correlates with advanced histopathological stage of colorectal adenocarcinoma, and the data from this study support the notion that EGFR expression may up-regulate EMMPRIN expression.

\section{References}

[1] A.B. Singh and R.C. Harris, Autocrine, paracrine and juxtacrine signaling by EGFR ligands, Cell. Signal. 17 (2005), 1183-1193.

[2] A. Hidalgo, P. Pina, G. Guerrero, M. Lazos and M. Salcedo, A simple method for the construction of a small format tissue arrays, J. Clin. Pathol. 56 (2003), 144-146.

[3] A.P. Dei Tos and I. Ellis, Assessing epidermal growth factor receptor expression in tumours: what is the value of current test methods? Eur. J. Cancer 41 (2005), 1383-1392.

[4] A. Wells, Molecules in focus EGF receptor, Int. J. Biochem. Cell Biol. 31 (1999), 637-643.

[5] B. Davidson, S. Konstantinovsky, S. Nielsen, H.P. Dong, A. Berner, M. Vyberg and R. Reich, Altered expression of metastasis-associated and regulatory molecules in effusions from breast cancer patients: a novel model for tumor progression, Clin. Cancer Res. 10 (2004), 7335-7346.

[6] B. Davidson, V. Givant-Horwitz, P. Lazarovici, B. Risberg, J.M. Nesland, C.G. Trope, E. Schaefer and R. Reich, Matrix metalloproteinases (MMP), EMMPRIN (extracellular matrix metalloproteinase inducer) and mitogen-activated protein kinases (MAPK): co-expression in metastatic serous ovarian carcinoma, Clin. Exp. Metastasis 20 (2003), 621-631.

[7] C. Biswas, Y. Zhang, R. DeCastro, H. Guo, T. Nakamura, H. Kataoka and K. Nabeshima, The human tumor cell-derived collagenase stimulatory factor (renamed EMMPRIN) is a member of the immunoglobulin superfamily, Cancer Res $\mathbf{5 5}$ (1995), 434-439.

[8] D.G. Rosen, X. Huang, M.T. Deavers, A. Malpica, E.G. Silva and J. Lium, Validation of tissue microarray technique in ovarian carcinoma, Mod. Pathol. 17 (2004), 790-797.

[9] D.S. Salomon, R. Brandt, F. Ciardiello and N. Normanno, Epidermal growth factor-related peptides and their receptors in human malignancies, Crit. Rev. Oncol. Haematol. 19 (1995), 183-232.

[10] E.E. Gabison, H.X. Thanh, A. Mauviel and S. Menashi, EMMPRIN/CD147, an MMP modulator in cancer development and tissue repair, Biochimie 87 (2005), 361-368.

[11] E.L. Rosenthal, S. Shreenivas, G.E. Peters, W.E. Grizzle, R. Desmond and C.L. Gladson, Expression of extracellular matrix metalloprotease inducer in laryngeal squamous cell carcinoma, Laryngoscope 113 (2003), 1406-1410.

[12] F. Caponigro, R. Formato, M. Caraglia, N. Normanno and R.V. Iaffaioli, Monoclonal antibodies targeting epidermal growth factor receptor and vascular endothelial growth factor with a focus on head and neck tumors, Curr. Opin. Oncol. 17 (2005), 212-217.
[13] H. Guo, G. Majmudar, T.C. Jensen, C. Biswas, B.P. Toole and M.K. Gordon, Characterization of the gene for human EMMPRIN, a tumor cell surface inducer of matrix metalloproteinases, Gene 220 (1998), 99-108.

[14] J. Jacquemier, C. Ginestier, F. Bertucci, E.C. Jauffret, J. Geneix and D. Birnbaum, Tissue microarrays: a powerful tool in transfer and quality control in oncology, Bull. Cancer 90 (2003), 31-38.

[15] J.P. Spano, R. Fagard, J.C. Soria, O. Rixe, D. Khayat and G. Milano. Epidermal growth factor receptor signaling in colorectal cancer: preclinical data and therapeutic perspectives, Ann. Oncol. 16 (2005), 189-194.

[16] K. Al-Kuraya, R. Simon and G. Sauter, Tissue microarrays for high-throughput molecular pathology, Ann. Saudi Med. 24 (2004), 169-174.

[17] K. Dalberg, E. Eriksson, U. Enberg, M. Kjellman, M. Backdahl and A. Gelatinase, Membrane type 1 matrix metalloproteinase, and extracellular matrix metalloproteinase inducer mRNA expression: correlation with invasive growth of breast cancer, World J. Surg. 24 (2000), 334-340.

[18] K. Muraoka, K. Nabeshima, T. Murayama, C. Biswas and M. Koono, Enhanced expression of a tumor-cell-derived collagenase-stimulatory factor in urothelial carcinoma; its usefulness as a tumor marker for bladder cancers, Int. J. Cancer 55 (1993), 19-26.

[19] K. Nabeshima, J. Suzumiya, M. Nagano, K. Ohshima, B. P. Toole, K. Tamura, H. Iwasaki and M. Kikuchi, Emmprin, a cell surface inducer of matrix metalloproteinases (MMPs), is expressed in T-cell lymphomas, J. Pathol. 202 (2004), 341351.

[20] M. Lim, T. Martinez, D. Jablons, R. Cameron, H. Guo, B. Toole, J.D. Li and C. Basbaum, Tumor-derived EMMPRIN (extracellular matrix metalloproteinase inducer) stimulates collagenase transcription through MAPK p38, FEBS Lett 441 (1998), 88-92.

[21] M. Mengel, H. Kreipe and R. von Wasielewski, Rapid and large-scale transition of new tumor biomarkers to clinical biopsy material by innovative tissue microarray systems, Appl. Immunohistochem. Mol. Morphol. 11 (2003), 261-268.

[22] N. Reimers, K. Zafrakas, V. Assmann, C. Egen, L. Riethdorf, S. Riethdorf, J. Berger, S. Ebel, F. Janicke, G. Sauter and K. Pantel, Expression of extracellular matrix metalloproteases inducer on micrometastatic and primary mammary carcinoma cells, Clin. Cancer Res. 10 (2004), 3422-3428.

[23] O.P. Kallioniemi, U. Wagner, J. Kononen and G. Sauter, Tissue microarray technology for high-throughput molecular profiling of cancer, Hum. Mol. Genet. 10 (2001), 657-662.

[24] R. Bianco, G. Daniele, F. Ciardiello and G. Tortora, Monoclonal antibodies targeting the epidermal growth factor receptor, Curr. Drug Targets 6 (2005), 275-287.

[25] R. Li, L. Huang, H. Guo and B.P. Toole, Basigin (murine EMMPRIN) stimulates matrix metalloproteinase production by fibroblasts, J. Cell. Physiol. 186 (2001), 371-379.

[26] S. Menashi, M. Serova, L. Ma, S. Vignot, S. Mourah and F. Calvo, Regulation of extracellular matrix metalloproteinase inducer and matrix metalloproteinase expression by amphiregulin in transformed human breast epithelial cells, Cancer Res 63 (2003), 7575-7580.

[27] T. Betsuyaku, M. Tanino, K. Nagai, Y. Nasuhara, M. Nishimura and R.M. Senior, Extracellular matrix metalloproteinase inducer is increased in smokers' bronchoalveolar lavage fluid, Am. J. Respir. Crit. Care Med. 168 (2003), 222227. 
[28] Z.D. Gu, K.N. Chen, M. Li, J. Gu and J.Y. Li, Clinical significance of matrix metalloproteinase-9 expression in esophageal squamous cell carcinoma, World J. Gastroenterol. 11 (2005), 871-874. 


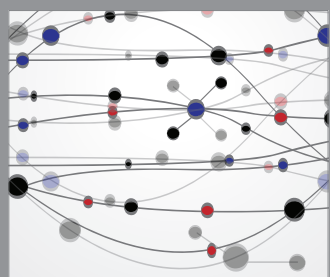

The Scientific World Journal
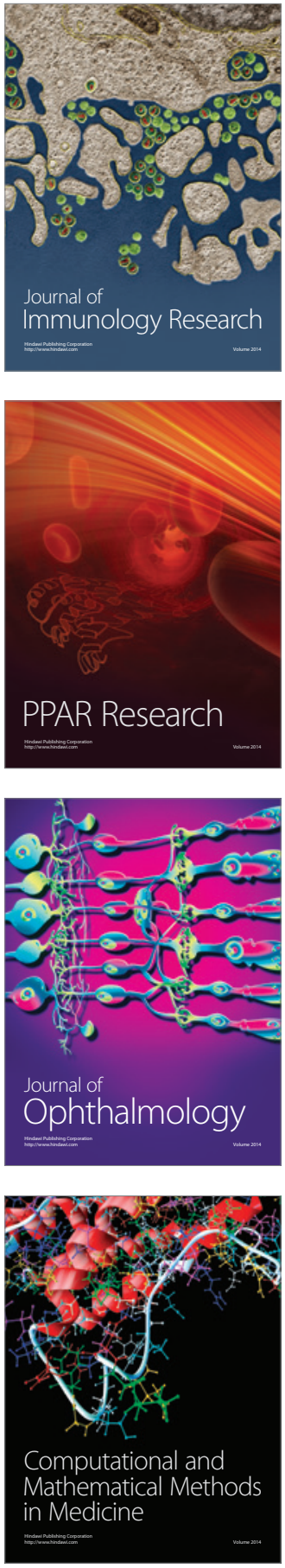

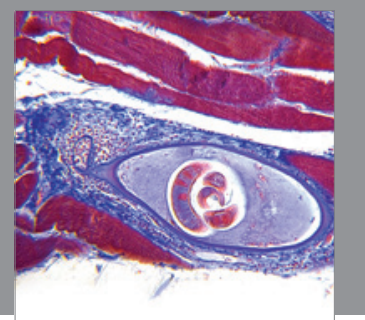

Gastroenterology

Research and Practice
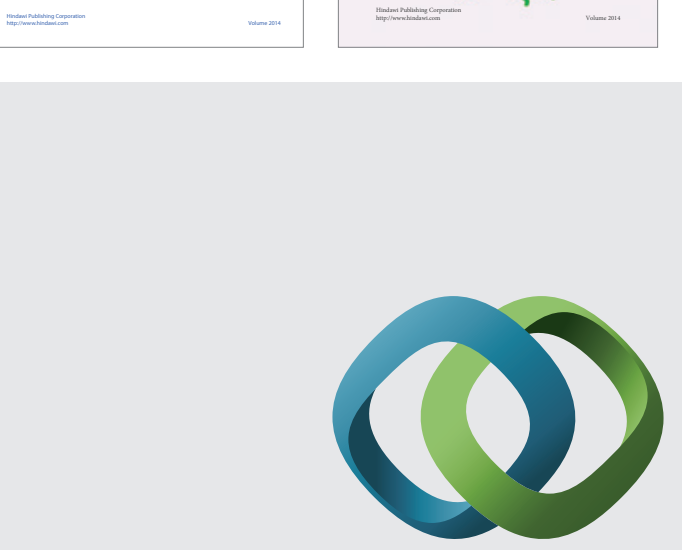

\section{Hindawi}

Submit your manuscripts at

http://www.hindawi.com
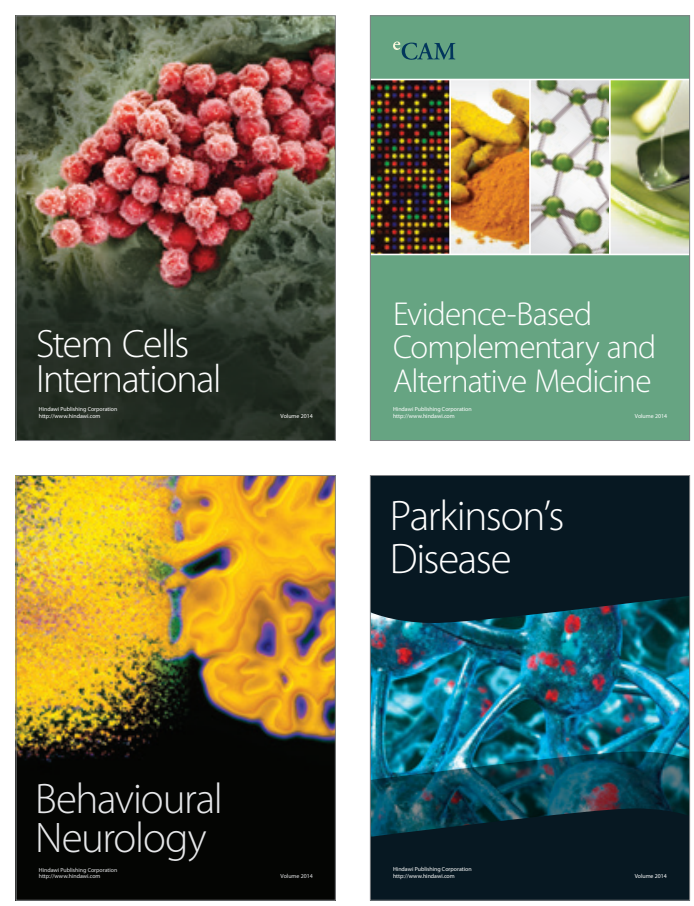

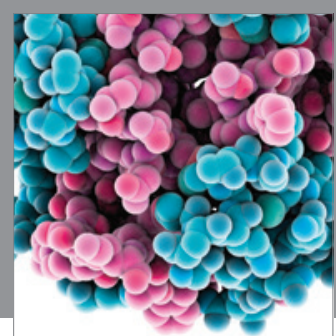

Journal of
Diabetes Research

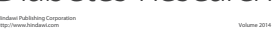

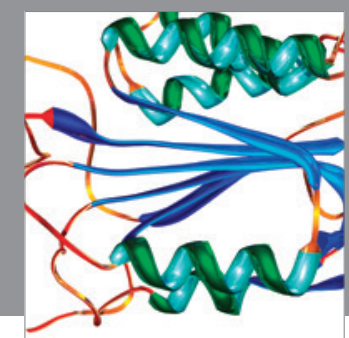

Disease Markers
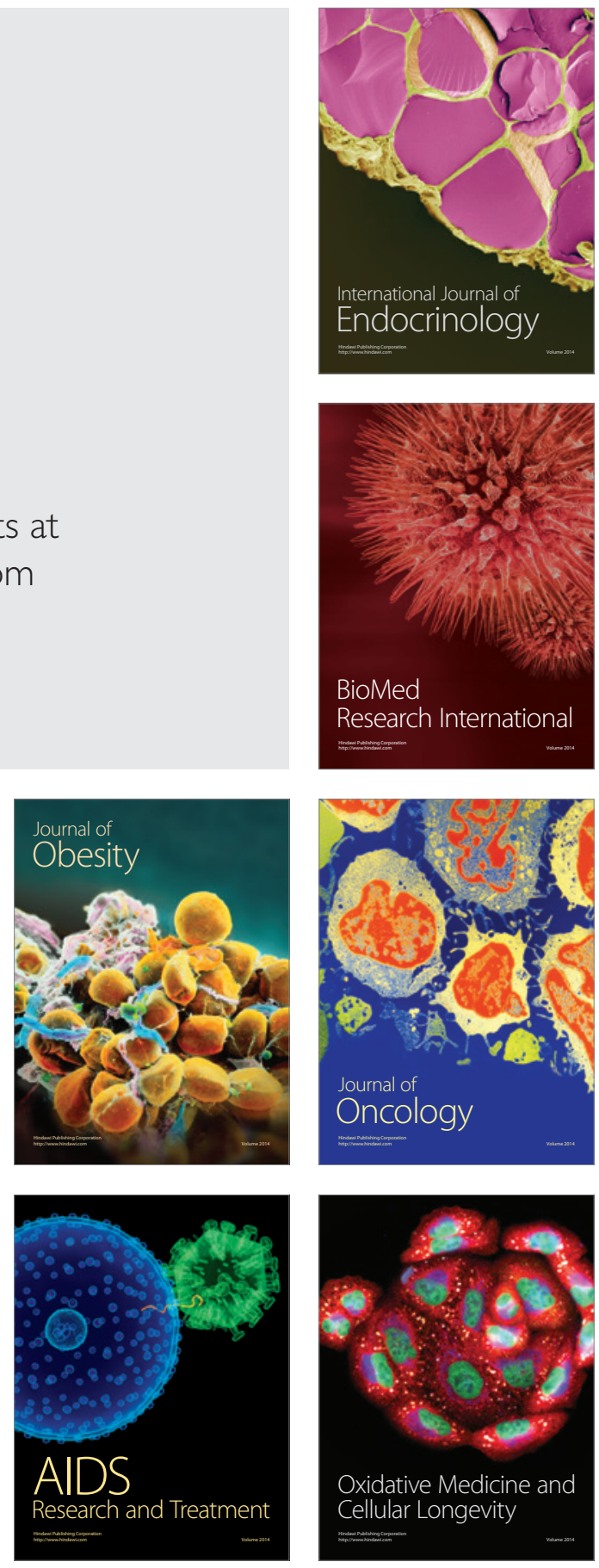